\title{
Pacific
}

Journal of

Mathematics

\section{SEMISIMPLICITY OF RESTRICTED ENVELOPING ALGEBRAS OF LIE SUPERALGEBRAS}

\author{
JEFFERY MARC BERGEN
}




\section{SEMISIMPLICITY OF RESTRICTED ENVELOPING ALGEBRAS OF LIE SUPERALGEBRAS}

\section{JEFFREY BERGEN}

Let $L=L_{0} \oplus L_{1}$ be a restricted Lie superalgebra over a field of characteristic $p>2$. We let $u(L)$ denote the restricted enveloping algebra of $L$ and we will be concerned with when $u(L)$ is semisimple, semiprime, or prime.

The structure of $u(L)$ is sufficiently close to that of a Hopf algebra that we will obtain ring theoretic information about $u(L)$ by first applying basic facts about finite dimensional Hopf algebras to Hopf algebras of the form $u(L) \# G$. Our main result along these lines is that if $u(L)$ is semisimple with $L$ finite dimensional, then $L_{1}=$ 0 . Combining this with a result of Hochschild, we will obtain a complete description of those finite dimensional $L$ such that $u(L)$ is semisimple.

In the infinite dimensional case, we will obtain various necessary conditions for $u(L)$ to be prime or semiprime.

Introduction. Let $L=L_{0} \oplus L_{1}$ be a restricted Lie superalgebra over a field $K$ of characteristic $p>2$. We let $u(L)$ denote the restricted enveloping algebra of $L$ and we will be concerned with when $u(L)$ is semisimple, semiprime, or prime.

When $L_{1} \neq 0, u(L)$ is not a Hopf algebra. However the structure of $u(L)$ is sufficiently close to that of a Hopf algebra that we can construct a skew group ring $u(L) \# G$ which is a Hopf algebra. We will obtain ring theoretic information about $u(L)$ by first applying basic facts about finite dimensional Hopf algebras to $u(L) \# G$. Our main result along these lines is

THEOREM. If $L$ is finite dimensional such that $u(L)$ is semisimple then $L_{1}=0$.

Combining this theorem with Hochschild's theorem [H] on the semisimplicity of $u\left(L_{0}\right)$, it easily follows that

COROLlaRY. If $L$ is finite dimensional then $u(L)$ is semisimple if and only if $L_{1}=0, L$ is abelian, and the pth power map on $L_{0}$ is injective. 
We begin in $\S 1$ with the definitions and terminology for restricted Lie superalgebras and their restricted enveloping algebras. In $\S 2$, we examine some of the basic properties of finite dimensional Hopf algebras and construct Hopf algebras of the form $u(L) \# G$. This construction not only yields ring theoretic information about $u(L)$, but also gives natural examples of finite dimensional noncommutative, noncocommutative Hopf algebras. In $\S 3$, in addition to the above theorem on the semisimplicity of $u(L)$, we find some necessary conditions for $u(L)$ to be prime or semiprime in the infinite dimensional case.

1. Definitions and terminology. We now introduce the terminology which will be used throughout this paper.

Definition. Suppose $L$ is a vector space over a field $K$ of characteristic $p>2$ which has a $K$-subspace decomposition $L=L_{0} \oplus L_{1}$. We say that $L$ is a restricted Lie superalgebra if there is a $K$-linear map [, ] and a $p$ th power map $L_{0} \rightarrow L_{0}$, denoted as [p], satisfying

(L1) $\left[L_{a}, L_{b}\right] \subseteq L_{a+b}$, where $a+b$ is computed modulo 2 ,

(L2) $[y, x]=-(-1)^{a b}[x, y]$ for all $x \in L_{a}$ and $y \in L_{b}$,

(L3) $(-1)^{a c}[x,[y, z]]+(-1)^{a b}[y,[z, x]]+(-1)^{b c}[z,[x, y]]=0$ for all $x \in L_{a}, y \in L_{b}$, and $z \in L_{c}$.

(R1) $(k x)^{[p]}=k^{p} x^{[p]}$ for all $k \in K$ and $x \in L_{0}$, and

(R2) $\left[x^{[p]}, y\right]=(\operatorname{ad} x)^{p}(y)$ for all $x \in L_{0}$ and $y \in L$,

(R3) $(x+y)^{[p]}=x^{[p]}+y^{[p]}+\sum_{i=1}^{p-1} s_{i}(x, y)$ for all $x, y \in L_{0}$ where $(\operatorname{ad} x)(y)=[x, y]$ and $i s_{i}$ is the coefficient of $\lambda^{i-1}$ in $(\operatorname{ad}(\lambda x+y))^{p-1}(x)$.

If $K$ has characteristic 3 , we also need to assume in the definition that $[[y, y], y]=0$, for all $y \in L_{1}$. For more details on Lie superalgebras, we refer the reader to [Sc]. $L$ admits a $K$-linear map $\sigma$ defined as $\left(x_{0}+x_{1}\right)^{\sigma}=x_{0}-x_{1}$, where $x_{i} \in L_{i}$. It is easy to see that $L_{0}=\left\{l \in L \mid l^{\sigma}=l\right\}, L_{1}=\left\{l \in L \mid l^{\sigma}=-l\right\}, \sigma^{2}=1$, and if $L_{1} \neq 0$ then $\sigma \neq 1$. We will often refer to the elements of $L_{0}$ and $L_{1}$ as the homogeneous elements of $L$. If $x, y$ are homogeneous then $\left[x^{\sigma}, y^{\sigma}\right]=[x, y]^{\sigma}$; therefore by the linearity of $[$,$] , it follows that$ $\left[x^{\sigma}, y^{\sigma}\right]=[x, y]^{\sigma}$ for all $x, y \in L$. Thus $\sigma$ is an automorphism of $L$.

There exists a unique largest $K$-algebra $u(L) \supseteq L$, such that $u(L$. is generated by $L$ with relations $x y-(-1)^{a b} y x=[x, y]$, for all $x \in L_{a}$ and $y \in L_{b}$, and $x^{p}=x^{[p]}$, for all $x \in L_{0}$. We call $u(L)$ the restricted enveloping algebra of $L$. The following analog of Jacobson's Theorem $[\mathbf{J}]$ on $u\left(L_{0}\right)$ asserts that $u(L)$ exists: 
JACOBSON'S THEOREM. Let $L$ be a restricted Lie superalgebra in char $p>2$ and let $C$ be a totally ordered basis for $L$ consisting of homogeneous elements. Then $u(L)$ has as a $K$-basis all ordered monomials

$$
b_{1}^{\beta_{1}} b_{2}^{\beta_{2}} \cdots b_{n}^{\beta_{n}}
$$

such that $b_{i} \in C, b_{1}<b_{2}<\cdots<b_{n}$, and such that $0 \leq \beta_{i}<p$ whenever $b_{i} \in L_{0}$ and $0 \leq \beta_{i} \leq 1$ whenever $b_{i} \in L_{1}$.

If $L_{0}$ is $n$-dimensional and $L_{1}$ is $m$-dimensional then Jacobson's Theorem implies that $u(L)$ has dimension $p^{n} 2^{m}$. The automorphism $\sigma$ of $L$ can be extended to an automorphism of $u(L)$ with $\sigma^{2}=1$. As a result, we can decompose $u(L)$ as $u(L)=U_{0} \oplus U_{1}$ where $U_{0}=$ $\left\{r \in u(L) \mid r^{\sigma}=r\right\}$ and $U_{1}=\left\{r \in u(L) \mid r^{\sigma}=-r\right\}$.

If $x \in L$ is homogeneous then the map $x: L \rightarrow L$ defined by $\alpha^{x}=[x, \alpha]$, for all $\alpha \in L$, extends to a map on all of $u(L)$. We call this map the superderivation induced by $x$ and satisfies the following properties:

(S1) For any $\alpha \in u(L)$,

$$
\alpha^{x}= \begin{cases}x \alpha-\alpha x & \text { if } x \in L_{0}, \\ x \alpha-\alpha^{\sigma} x & \text { if } x \in L_{1},\end{cases}
$$

(S2) For any $\alpha, \beta \in u(L)$,

$$
(\alpha \beta)^{x}=\left\{\begin{array}{ll}
\alpha^{x} \beta+\alpha \beta^{x} & \text { if } x \in L_{0}, \\
\alpha^{x} \beta+\alpha^{\sigma} \beta^{x} & \text { if } x \in L_{1}
\end{array} .\right.
$$

A $K$-subspace $B$ of $u(L)$ is called $L$-invariant if $B^{x} \subseteq B$ for all homogeneous $x \in L$, and is called homogeneous if $B^{\sigma}=B$. If $B$ is homogeneous then $B$ can be decomposed as $B=B_{0} \oplus B_{1}$ where $B_{0}=\left\{b \in B \mid b^{\sigma}=b\right\}$ and $B_{1}=\left\{b \in B \mid b^{\sigma}=-b\right\}$. Observe that if $J$ is a homogeneous ideal of $u(L)$ then (S1) implies that $J$ must also be $L$-invariant. A $K$-subspace $I$ of $L$ will be called a Lie superideal of $L$ if $I$ is $L$-invariant and homogeneous. If, in addition, $I_{0}$ is closed under the $p$ th power map we say that $I$ is a restricted Lie superideal.

We will need the following lemma in $\S 3$.

LEMMA 1.1. Let $A$ be an L-invariant, homogeneous subspace of $u(L)$. Then $A u(L)=u(L) A$.

Proof. In order to prove that $A u(L) \subseteq u(L) A$ it suffices, by Jacobson's Theorem, to show that $a x_{1} \cdots x_{n} \in u(L) A$ for all homogeneous $a \in A$ and homogeneous $x_{i} \in L$. We proceed by induction 
on $n$; the result is clear when $n=0$. Therefore we may assume that $a x_{1} \cdots x_{n}=\sum_{i=0}^{m} t_{i} b_{i}$ where $t_{i} \in u(L)$ and the $b_{i}$ are homogeneous elements of $A$. Now if $x \in L$ is homogeneous then, by (S1), either $b_{i}^{x}=x b_{i}-b_{i} x$ or $b_{i}^{x}=x b_{i}+b_{i} x$, for every $i$. Since $b_{i}^{x} \in A$, both of the above cases imply that $b_{i} x \in u(L) A$. Hence $t_{i} b_{i} x \in u(L) A$ and so, $a x_{1} \cdots x_{n} x=\sum_{i=0}^{m} t_{i} b_{i} x \in u(L) A$. An analogous argument shows that $u(L) A \subseteq A u(L)$.

2. Hopf algebras. Throughout this section, $H$ will be a Hopf algebra over a field $K$ of arbitrary characteristic. We let $\Delta: H \rightarrow H \otimes H$ be the comultiplication, $\varepsilon: H \rightarrow K$ the counit, and $S: H \rightarrow H$ the antipode. For a more thorough introduction to Hopf algebra terminology, we recommend [A] or [Sw]. For Lie algebras and restricted Lie algebras $L_{0}$, it is well known that both the ordinary enveloping algebra $U\left(L_{0}\right)$ and the restricted enveloping algebra $u\left(L_{0}\right)$, are Hopf algebras where $\Delta(x)=x \otimes 1+1 \otimes x, \varepsilon(x)=0$, and $S(x)=-x$, for all $x \in L_{0}$.

Now suppose $L=L_{0} \oplus L_{1}$ is a Lie superalgebra or a restricted Lie superalgebra, then if $y \in L_{1}$ the superderivation induced by $y$ satisfies (S2) on the ordinary and restricted enveloping algebras $U(L)$ and $u(L)$. Therefore if $\alpha, \beta \in U(L)$ or $u(L)$ then $(\alpha \beta)^{y}=\alpha^{y} \beta+$ $\alpha^{\sigma} \beta^{y}$. However, every Hopf algebra acts on itself via left and right adjoint actions. For all $\alpha, \beta, h \in H$ these adjoint actions satisfy

$$
(\alpha \beta)^{h}=\sum_{(h)} \alpha^{h_{1}} \beta^{h_{2}}
$$

where $\sum_{(h)} h_{1} \otimes h_{2}$ is the comultiplication of $h$ in the sigma notation of Heyneman and Sweedler.

Contrasting (*) with (S2), we see that for $U(L)$ or $u(L)$ to be contained in a Hopf algebra, we would need $\Delta(y)=y \otimes 1+\sigma \otimes y$. In particular, if $L_{1} \neq 0$ then neither $U(L)$ or $u(L)$ is a Hopf algebra. However the formula $\Delta(y)=y \otimes 1+\sigma \otimes y$ does indicate a way to construct a Hopf algebra by essentially adjoining $\sigma$ to $U(L)$ or $u(L)$.

If $L_{1} \neq 0$ then $\sigma$ induces an automorphism of order 2 of both $U(L)$ and $u(L)$. Letting $G$ be the group $\{1, \sigma\}$, we can form the skew group rings $U(L) \# G$ and $u(L) \# G$. In both of these rings, $x \sigma=\sigma x$ and $y \sigma=-\sigma y$, for all $x \in L_{0}$ and $y \in L_{1} . U(L) \# G$ and $u(L) \# G$ can both be made into Hopf algebras by defining $\Delta, \varepsilon$, and 
$S$ as follows:

$$
\begin{aligned}
& \Delta(x)=x \otimes 1+1 \otimes x, \quad \varepsilon(x)=0, \quad S(x)=-x, \quad \text { for all } x \in L_{0}, \\
& \Delta(y)=y \otimes 1+\sigma \otimes y, \quad \varepsilon(y)=0, \quad S(y)=y \sigma, \quad \text { for all } y \in L_{1}, \\
& \Delta(\sigma)=\sigma \otimes \sigma, \quad \varepsilon(\sigma)=1, \quad S(\sigma)=\sigma .
\end{aligned}
$$

It is not difficult, but somewhat tedious, to check that $U(L) \# G$ and $u(L) \# G$ satisfy all of the Hopf algebra axioms. The details for this construction, as well as for more general Hopf algebra constructions, can be found in [R, Theorem 1]. We note that both $U(L) \# G$ and $u(L) \# G$ are noncommutative as well as noncocommutative. We record these observations as

Proposition 2.1. Let $L=L_{0} \oplus L_{1}$ be either a Lie superalgebra or a restricted Lie superalgebra with $L_{1} \neq 0$. If $U(L)$ and $u(L)$ are the enveloping algebra and the restricted enveloping algebras of $L$, then the skew group rings $U(L) \# G$ and $u(L) \# G$ are noncommutative, noncocommutative Hopf algebras, where $G=\{1, \sigma\}$.

Since there is interest in the construction of finite dimensional noncommutative, noncocommutative Hopf algebras, we now use Jacobson's Theorem and Proposition 2.1 to observe that

COROLLARY 2.2. If $L$ is a restricted Lie superalgebra over a field of characteristic $p>2$ such that $L_{0}$ is $n$-dimensional and $L_{1} \neq 0$ is $m$ dimensional, then $u(L) \# G$ is a noncommutative, noncocommutative Hopf algebra of dimension $p^{n} 2^{m+1}$.

In $\S 3$, we will use fundamental facts about the structure of finite dimensional Hopf algebras to gain information about the structure of $u(L) \# G$. This will enable us to obtain ring theoretic information about $u(L)$. The Hopf algebra facts we will need are

Proposition 2.3. Let $H$ be a finite dimensional Hopf algebra. Then

(i) if $\omega$ is the kernel of $\varepsilon$, then the right annihilator of $\omega$ is a one-dimensional ideal of $H$ known as the left integral and denoted as $\int_{H}$.

(ii) $H$ is semisimple if and only if $\varepsilon\left(\int_{H}\right) \neq 0$.

(iii) If $M$ is a Hopf subalgebra of $H$ with left integral $\int_{M}$ then there exists an $h \in H$ such that $\int_{H}=\int_{M} h$.

(iv) $H$ is semisimple if and only if every Hopf subalgebra of $H$ is also semisimple. 
Parts (i) and (ii) are results of Larson and Sweedler [LS]. Part (iii) follows from the freeness result of Nichols-Zoeller [NZ]. Briefly, if $M$ is a Hopf subalgebra of $H$ then $H$ is free as a left $M$-module with basis $\left\{h_{i}\right\}$. Let $t \in \int_{H}$; then there exist $m_{i} \in M$ such that $t=$ $\sum_{i} m_{i} h_{i}$. If $k \in M \cap \omega$ then $0=k t=\sum_{i} k m_{i} h_{i}$. By (i), each $m_{i} \in$ $\int_{M}$ and there exist $\alpha_{i} \in K$ and $m \in \int_{M}$ such that $m_{i}=\alpha_{i} m$. Thus $t=m\left(\sum_{i} \alpha_{i} h_{i}\right)$ as required. For part (iv), let $M$ be a Hopf subalgebra of $H$ and let $t$ and $m$ be as in the previous argument. Now if $H$ is semisimple then, by (ii), we may assume that $\varepsilon(t) \neq 0$. However $\varepsilon$ is an algebra homomorphism, hence $0 \neq \varepsilon(t)=\varepsilon(m) \varepsilon\left(\sum_{i} \alpha_{i} h_{i}\right)$ and so, $\varepsilon(m) \neq 0$. Thus $M$ is also semisimple.

3. The main results. We can now prove the main result of this paper.

THEOREM 3.1. Let $L$ be a finite dimensional restricted Lie superalgebra. If $u(L)$ is semisimple then $L_{1}=0$.

Proof. If there exists some non-zero $y \in L_{1}$, let $A$ be the restricted supersubalgebra of $L$ generated by $y$. Letting $x=[y, y]$, it follows that $A=A_{0} \oplus A_{1}$, where $A_{1}=\langle y\rangle$ and either $A_{0}=0$ or $A_{0}=$ $\left\langle x, x^{[p]}, x^{\left[p^{2}\right]}, \ldots, x^{\left[p^{n}\right]}\right\rangle$ for some natural number $n$. A result of Fisher-Montgomery [FM] states that if $G$ is a finite group and if $R$ is a ring with no $|G|$-torsion then $R$ is semiprime if and only if $R \# G$ is semiprime. Since in the finite dimensional case being semisimple is equivalent to being semiprime, we can apply the result of [FM] to the Hopf algebra $u(L) \# G$ of Corollary 2.2 to conclude that if $u(L)$ is semisimple then $u(L) \# G$ is also semisimple. The skew group ring $H=u(A) \# G$ is a Hopf subalgebra of $u(L) \# G$; thus by Proposition 2.3(iv), $H$ is also semisimple. Thus, by Proposition 2.3(ii), there exists a $t \in \int_{H}$ such that $\varepsilon(t) \neq 0$.

$H$ is a free left $u\left(A_{0}\right)$-module; in particular

$$
H=u\left(A_{0}\right) \oplus u\left(A_{0}\right) y \oplus u\left(A_{0}\right) \sigma \oplus u\left(A_{0}\right) y \sigma .
$$

Therefore there exist $a_{1}, a_{2}, a_{3}, a_{4} \in u\left(A_{0}\right)$ such that $t=a_{1}+a_{2} y+$ $a_{3} \sigma+a_{4} y \sigma$. Furthermore $y$ commutes with $u\left(A_{0}\right), \varepsilon(y)=0$, and $x=2 y^{2}$; therefore we have

$$
0=y t=a_{1} y+\frac{1}{2} a_{2} x+a_{3} y \sigma+\frac{1}{2} a_{4} x \sigma .
$$

Since $a_{2} x, a_{4} x \in u\left(A_{0}\right)$, the direct sum decomposition of $H$ implies that $a_{1}=a_{3}=0$. Therefore $t=a_{2} y+a_{4} y \sigma$. Now applying the 
homomorphism $\varepsilon$ yields $\varepsilon(t)=\varepsilon\left(a_{2} y+a_{4} y \sigma\right)=\varepsilon(y) \varepsilon\left(a_{2}+a_{4} \sigma\right)=0$, a contradiction. Thus $L_{1}=0$ thereby proving the result.

In [H], Hochschild shows that if $L_{0}$ is finite dimensional then $u\left(L_{0}\right)$ is semisimple if and only if $L_{0}$ is abelian and the $p$ th power map is injective. Combining Hochschild's result with Theorem 3.1 we obtain

COROLlaRY 3.2. Let $L$ be a finite dimensional restricted Lie superalgebra. $u(L)$ is semisimple if and only if $L_{1}=0, L_{0}$ is abelian, and the pth power map on $L_{0}$ is injective.

In order to move on to the infinite dimensional case, we first need to prove a stronger version of Theorem 3.1. We will essentially be showing that although $u(L)$ is not a Hopf algebra, it does contain an ideal analogous to the left integral in a Hopf algebra.

THeOREM 3.3. If $L$ is finite dimensional with $L_{1} \neq 0$ then $u(L)$ contains a one-dimensional homogeneous ideal $J$ such that $J^{2}=0$ and $J$ is the right annihilator in $u(L)$ of $L$.

Proof. In the finite dimensional Hopf algebra $H=u(L) \# G$, choose a non-zero $t \in \int_{H}$. Therefore there exist $a, b \in u(L)$ such that $t=a+b \sigma$. By Proposition 2.3(i), if $l \in L$ then $l t=0$ and $t l=\alpha t$, for some $\alpha \in K$. Thus $l t=l a+l b \sigma=0$ and $t l=a l+b l^{\sigma} \sigma=$ $\alpha(a+b \sigma)$. As a result, $L a=0$ and $a L \subseteq K a$, and so $K a$ is an ideal of $u(L)$ which annihilates $L$ on the right. Also by Proposition 2.3(i), $(1-\sigma) t=0$ and $t(1-\sigma)=\beta t$, for some $\beta \in K$. Thus $(1-\sigma)(a+b \sigma)=\left(a-b^{\sigma}\right)+\left(-a^{\sigma}+b\right) \sigma=0$ and $(a+b \sigma)(1-\sigma)=$ $(a-b)+(-a+b) \sigma=\beta(a+b \sigma)$. Thus $a^{\sigma}=b$ and $a-b=\beta a$ which imply that $a^{\sigma}=\gamma \alpha$ for some $\gamma \in K$. Since $\sigma^{2}=1$, it follows that $\gamma^{2}=1$, and thus $\gamma= \pm 1$. As a result $a$ is homogeneous, and hence the ideal $K a$ is certainly homogeneous. Furthermore, since $t=a+a^{\sigma} \sigma$ and $t \neq 0$, it follows that $a \neq 0$. Thus the ideal $K a$ is indeed one-dimensional.

Now suppose $c \in U(L)$ such that $L c=0$. Let $d=c+c^{\sigma}$ and $e=c-c^{\sigma}$; clearly $L d=L e=0$. Furthermore $(1-\sigma)(d+d \sigma)=$ $(1-\sigma)(e-e \sigma)=0$; thus both $d+d \sigma$ and $e-e \sigma$ belong to $\int_{H}$. Therefore, by Proposition 2.3(i), there exist $\alpha_{1}, \alpha_{2} \in K$ such that $d+d \sigma=\alpha_{1}(a+b \sigma)$ and $e-e \sigma=\alpha_{2}(a+b \sigma)$. Hence $c+c^{\sigma}=\alpha_{1} a$ and $c-c^{\sigma}=\alpha_{2} a$. It now easily follows that $c \in K a$, thus $K a$ is the right annihilator of $L$ in $u(L)$. 
Finally, it suffices to show that $a^{2}=0$. We know that $a^{\sigma}= \pm a$, and we will handle the two cases separately. If $a^{\sigma}=a$ then $t=a+a \sigma$; hence $\varepsilon(t)=2 \varepsilon(a)$. Since $u(L) \# G$ is not semisimple $\varepsilon(t)=0$; thus $\varepsilon(a)=0$, and so $a \in u(L) L$. However $L a=0$ and it follows that $a^{2}=0$. On the other hand, if $a^{\sigma}=-a$ then $t=a-a \sigma$; hence $t a=a^{2}-a \sigma a=a^{2}+a^{2} \sigma$. However, there exists $\alpha \in K$ such that $t a=\alpha t=\alpha(a-a \sigma)$. As a result $a^{2}=\alpha a$ and $a^{2}=-\alpha a$; hence $a^{2}=0$ as required.

The existence of a "left integral" in $u(L)$ might turn out to be quite useful in attempting to study the invariants of restricted superalgebras as the action of the element $a$ in the proof of Theorem 3.3 would serve as a trace map. As we move to the case where $L$ is infinite dimensional, instead of being concerned with semisimplicity we will primarily be concerned with when $u(L)$ is prime or semiprime. We continue with

EXAMPLE 3.4. Let $L=L_{0} \oplus L_{1}$ where $L_{0}=\left\langle x, x^{[p]}, x^{\left[p^{2}\right]}, \ldots\right\rangle$ and $L_{1}=\langle y\rangle$ where the only non-trivial bracket relation is $[y, y]=$ $x$. Therefore $u\left(L_{0}\right) \cong K[x]$, the polynomial ring in one variable, and $u(L) \cong K[x, y] /\left(x-2 y^{2}\right)$. Thus $u(L) \cong K[y]$, also a polynomial ring in one variable. Hence $u(L)$ is both prime and semisimple.

The above example shows that in the infinite dimensional case $u(L)$ can be prime or semiprime even if $L_{1} \neq 0$. However we will now see that if $u(L)$ is semiprime with $L_{1} \neq 0$ then $L_{0}$ must be infinite dimensional.

THEOREM 3.5. Let $L$ be a restricted Lie superalgebra such that $u(L)$ is semiprime. If $L_{1} \neq 0$ then $L_{0}$ must be infinite dimensional.

Proof. Since every element of $u(L)$ is a linear combination of products of homogeneous elements, $u(L)$ acts on $L$ as sums and compositions of superderivations. If $0 \neq y \in L$, let $A$ denote the image of $y$ under the action of $u(L)$. It is easy to see that $A$ is homogeneous and is the smallest Lie superideal of $L$ containing $y$. We claim that if $L_{0}$ is finite dimensional then $A$ is also finite dimensional. To this end, note that $L^{y} \subseteq L_{0}^{y} \oplus L_{1}^{y} \subseteq L_{0}^{y} \oplus L_{0}$; thus if $L_{0}$ is finite dimensional then so is $L^{y}$. Therefore if $M=\{m \in L \mid[m, y]=0\}$ then $M$ is a homogeneous $K$-subspace of finite codimension in $L$. As a result there exists an ordered basis $X \dot{\cup} Y$ for $L$ such that $X<Y, Y$ is a basis for $M$, and $X$ is finite dimensional. By Jacobson's Theorem, if $\eta$ is a basis monomial of $u(L)$ then the image of $y$ under 
$\eta$ is 0 unless $\eta$ consists solely of elements from $X$. Since there are only a finite number of such $\eta$, the image of $y$ under $u(L)$ is finite dimensional and so, $A$ is finite dimensional.

$A=A_{0} \oplus A_{1}$ where $A_{0}$ is a Lie ideal of $L_{0}$; however $A_{0}$ need not be restricted. Let $\bar{A}_{0}$ be the span over $K$ of $\left\{a^{\left[p^{m}\right]} \in L_{0} \mid a \in A_{0}\right.$ and $m \geq 0\}$. Then by (R2) and (R3), $\bar{A}_{0}$ is restricted with $\left[\bar{A}_{0}, L\right] \subseteq$ $\left[A_{0}, L\right]$. Since $\bar{A}_{0}$ is finite dimensional, if we let $\bar{A}=\bar{A}_{0} \oplus A_{1}$ then $\bar{A}$ is a finite dimensional restricted Lie superideal of $L$.

By Theorem 3.3, the right annihilator of $\bar{A}$ in $u(\bar{A})$ is a onedimensional ideal $K a$, where $a$ is homogeneous and $a^{2}=0$. However, by (S2) if $x \in L$ is homogeneous then $0=(\bar{A} a)^{x}=(\bar{A})^{x} a+\bar{A} a^{x}$ or $0=(\bar{A} a)^{x}=(\bar{A})^{x} a+(\bar{A})^{\sigma} a^{x}$. In either case, since $\bar{A}$ is homogeneous and $L$-invariant, we have $a^{x} \in u(\bar{A})$ and $\bar{A} a^{x}=0$. Thus $a^{x} \in K a$, and hence $K a$ is an $L$-invariant homogeneous subspace of $L$. Now, by Lemma $1.1, a u(L)=u(L) a$ and so, $(a u(L))^{2}=$ $u(L) a^{2} u(L)=0$. As a result $a u(L)$ is a nilpotent ideal of $u(L)$; hence $u(L)$ is not semiprime.

The proof of Theorem 3.5 actually shows that if $I$ is a restricted Lie superideal of $L$ and if $J$ is the right annihilator of $I$ in $u(I)$, then $J u(L)=u(L) J$. In light of Theorem 3.5 and this observation we now have

COROllaRy 3.6. (i) If $u(L)$ is prime, then $L$ contains no non-zero finite dimensional restricted Lie superideals.

(ii) If $u(L)$ is semiprime and if $I$ is a finite dimensional restricted Lie superideal of $L$ then $u(I)$ is semisimple, $I \subseteq L_{0}$, and $\left[I, L_{1}\right]=0$.

(iii) If $u(L)$ is prime then $L_{0}$ must be infinite dimensional.

Proof. Let $I$ be a non-zero restricted Lie superideal of $L$ and $J$ the right annihilator of $I$ in $u(I)$. Thus $0=I(J u(L))=I(u(L) J)$. By Theorem 3.3, $J \neq 0$ and so, $u(L)$ is not prime. Furthermore if $J^{2}=0$ then $(J u(L))^{2}=u(L) J^{2} u(L)=0$. However, if $u(I)$ is not semiprime then, by Theorem $3.3, J^{2}=0$. Hence if $u(L)$ is semiprime then so is $u(I)$. Now, by Theorem 3.1, if $u(I)$ is semiprime the $I=I_{0} \subseteq L_{0}$ and clearly $\left[I, L_{1}\right] \subseteq I \cap L_{1}=0$. Finally, if $u(L)$ is prime then when $L_{1}=0, L_{0}$ must be infinite dimensional by Proposition 2.3(i), and when $L_{1} \neq 0, L_{0}$ must be infinite dimensional by Theorem 3.5 .

We continue with 
EXAMPLE 3.7. Let $L=L_{0} \oplus L_{1}$ where $L_{0}=\left\langle x, x^{[p]}, x^{\left[p^{2}\right]}, \ldots\right\rangle$, $L_{1}=\left\langle y_{1}, y_{2}, y_{3}, \ldots\right\rangle$ and the only non-trivial bracket relations are $\left[x^{\left[p^{n}\right]}, y_{i}\right]=y_{i+p^{n}}$, for all $i \geq 1$ and $n \geq 0$. There are several ways to see that $u(L)$ is prime. First, $u(L)$ is isomorphic to the differential operator ring $E[t, \delta]$, where $E$ is the infinite dimensional Grassmann algebra on $\left\{y_{i}\right\}$, and $\delta$ is the derivation $y_{i}^{\delta}=y_{i+1}$. This differential operator ring was shown to be prime in [BMP]. Another approach is to let $\mathbb{D}_{L}=\{l \in L \mid[L, l]$ is finite dimensional $\}$; in this example it is easy to see that $\mathbb{D}_{L}=0$. However, in [BP] it is shown that if $\mathbb{D}_{L}=0$ then $u(L)$ is prime.

In Examples 3.5 and 3.7, $u(L)$ is prime. However in Example 3.7, $\left[L_{1}, L_{1}\right]=0$ and $L_{1}$ is infinite dimensional. We now show that if $\left[L_{1}, L_{1}\right]=0$ then $L_{1}$ must be infinite dimensional for $u(L)$ to be semiprime.

COROLlary 3.8. If $u(L)$ 'is semiprime with $L_{1} \neq 0$ and $\left[L_{1}, L_{1}\right]=$ 0 then both $L_{0}$ and $L_{1}$ must be infinite dimensional.

Proof. By Theorem 3.5, $L_{0}$ must be infinite dimensional. Now if $\left[L_{1}, L_{1}\right]=0$ then $L_{1}$ is a homogeneous $L$-invariant subspace of $L$. Thus, by Lemma 1.1, $L_{1} u(L)=u(L) L_{1}$. If $L_{1}$ is $n$-dimensional, then the product of any $n+1$ elements of $L_{1}$ is zero in $u(L)$. Therefore $L_{1} u(L)$ is a nilpotent ideal of $u(L)$ as $\left(L_{1} u(L)\right)^{n+1}=$ $u(L) L_{1}^{n+1} u(L)=0$. As a result, if $u(L)$ is semiprime then $L_{1}$ must be infinite dimensional.

The flavor of many of the results in this paper is that $L_{1}$ being non-zero is, in some sense, an obstruction to $u(L)$ being prime or semiprime. We conclude this paper with an example showing that this is not necessarily the case. More precisely, in our example $u(L)$ will be prime even though $u\left(L_{0}\right)$ is not.

EXAMPLE 3.9. We slightly enlarge the Lie superalgebra from Example 3.7. We let $L=L_{0} \oplus L_{1}$ where $L_{0}=\left\langle z, x, x^{[p]}, x^{\left[p^{2}\right]}, \ldots\right\rangle$, $L_{1}=\left\langle y_{1}, y_{2}, y_{3}, \ldots\right\rangle$ and the only non-trivial relations are those of Example 3.7 along with $z^{[p]}=z$ and $\left[z, y_{i}\right]=y_{i}$, for all $i \geq 1$. It is again easy to check that $\mathbb{D}_{L}=0$, thus, by the result in $[\mathbf{B P}], u(L)$ is prime. However, $u\left(L_{0}\right)$ is not prime since it is commutative, but not a domain as $\left(z^{p-1}-1\right) z=0$. 


\section{REFERENCES}

[A] E. Abe, Hopf Algebras, Cambridge University Press, Cambridge-New York, 1980.

[BMP] J. Bergen, S. Montgomery and D. S. Passman, Radicals of crossed products of enveloping algebras, Israel J. Math., 59 (1987), 167-184.

[BP] J. Bergen and D. S. Passman, Delta methods in enveloping algebras of Lie superalgebras, Trans. Amer. Math. Soc., 334 (1992), 259-280.

[FM] J. W. Fisher and S. Montgomery, Semiprime skew group rings, J. Algebra, 52 (1978), 241-247.

[H] G. P. Hochschild, Representations of restricted Lie algebras of characteristic $p$, Proc. Amer. Math. Soc., 5 (1954), 603-605.

[J] N. Jacobson, Lie Algebras, Interscience, New York-London, 1962.

[LS] R. G. Larson and M. E. Sweedler, An associative orthogonal bilinear form for Hopf algebras, Amer. J. Math., 91 (1969), 75-94.

[NZ] W. D. Nichols and M. Bettina Zoeller, A Hopf algebra freeness theorem, Amer. J. Math., 111 (1989), 381-385.

[R] D. Radford, The structure of Hopf algebras with a projection, J. Algebra, 92 (1985), 322-347.

[Sc] M. Scheunert, The Theory of Lie Superalgebras, Lecture Notes in Mathematics, vol. 716, Springer-Verlag, Berlin, 1979.

[Sw] M. E. Sweedler, Hopf Algebras, Benjamin, New York, 1969.

Received August 25, 1991. Research supported in part by National Security Agency Grant MSP08491.

DePaul University

Chicago, IL 60614-8250 



\title{
PACIFIC JOURNAL OF MATHEMATICS
}

Founded by

\author{
E. F. BeCKenBACH (1906-1982) F. Wolf (1904-1989)
}

\section{EDITORS}

Sun-Yung A. Chang

(Managing Editor)

University of California

Los Angeles, CA 90024-1555

chang@math.ucla.edu

\section{F. Michael Christ}

University of California

Los Angeles, CA 90024-1555

christ@math.ucla.edu

Herbert Clemens

University of Utah

Salt Lake City, UT 84112

clemens@math.utah.edu
THOMAS ENRIGHT

University of California, San Diego

La Jolla, CA 92093

tenright@ucsd.edu

Nicholas ERCOLANI

University of Arizona

Tucson, AZ 85721

ercolani@math.arizona.edu

R. FINN

Stanford University

Stanford, CA 94305

finn@gauss.stanford.edu

VAUghan F. R. Jones

University of California

Berkeley, CA 94720

vfr@math.berkeley.edu
STEVEN KERCKHOFF

Stanford University

Stanford, CA 94305

spk@gauss.stanford.edu

Martin ScharLemanN University of California Santa Barbara, CA 93106 mgscharl@math.ucsb.edu

Harold Stark

University of California, San Diego La Jolla, CA 92093

V. S. VARADARAJAN University of California

Los Angeles, CA 90024-1555

vsv@math.ucla.edu

\section{SUPPORTING INSTITUTIONS}

UNIVERSITY OF ARIZONA

UNIVERSITY OF BRITISH COLUMBIA

CALIFORNIA INSTITUTE OF TECHNOLOGY

UNIVERSITY OF CALIFORNIA

UNIVERSITY OF MONTANA

UNIVERSITY OF NEVADA, RENO

NEW MEXICO STATE UNIVERSITY

OREGON STATE UNIVERSITY
UNIVERSITY OF OREGON

UNIVERSITY OF SOUTHERN CALIFORNIA

STANFORD UNIVERSITY

UNIVERSITY OF HAWAII

UNIVERSITY OF UTAH

WASHINGTON STATE UNIVERSITY

UNIVERSITY OF WASHINGTON 


\title{
PACIFIC JOURNAL OF MATHEMATICS
}

\author{
Volume $162 \quad$ No. $1 \quad$ January 1994
}

Semisimplicity of restricted enveloping algebras of Lie superalgebras $\quad 1$

JEFFERY MARC BERGEN

A classification of certain 3-dimensional conformally flat Euclidean hypersurfaces

OSCAR J. GARAY

Braided groups of Hopf algebras obtained by twisting

D. GUREVICH and SHAHN MAJID

Flat connections, geometric invariants and the symplectic nature of the 45 fundamental group of surfaces

K. GURUPRASAD

Spin models for link polynomials, strongly regular graphs and Jaeger's 57 Higman-Sims model

PIERRE DE LA HARPE

On the uniqueness of representational indices of derivations of $C^{*}$-algebras

EDWARD KISSIN

On infinitesimal behavior of the Kobayashi distance

MYUNG YULL PANG

Vertex operator construction of standard modules for $A_{n}^{(1)}$

MIRKO PRIMC

Bergman and Hardy spaces with small exponents

KEHE ZHU 